\title{
A planar ion trap chip with integrated structures for an adjustable magnetic field gradient
}

\author{
P. J. Kunert, D. Georgen, L. Bogunia, M. T. Baig, M. A. Baggash ${ }^{\dagger}$, M. Johanning, and Ch. Wunderlich \\ Naturwissenschaftlich-Technische Fakultät, Department Physik, Universität Siegen, 57068 Siegen, Germany \\ e-mail: wunderlich@physik.uni-siegen.de
}

Received: date / Revised version: date

\begin{abstract}
We present the design, fabrication, and characterization of a segmented surface ion trap with integrated current carrying structures. The latter produce a spatially varying magnetic field necessary for magnetic gradient induced coupling between ionic effective spins. We demonstrate trapping of strings of ${ }^{1} 72 \mathrm{Yb}^{+}$ions, characterize the performance of the trap and map magnetic fields by radio frequency-optical double resonance spectroscopy. In addition, we apply and characterize the magnetic gradient and demonstrate individual addressing in a string of three ions using RF radiation.
\end{abstract}

\section{Introduction}

Cold trapped ions have been established as a benchmark system in quantum information science and were used to show a variety of first proof of principle demonstrations [1,2] in this field. When scaling up the number of qubits or ions, a widely favored solution to limit detrimental effects of decoherence is to split the entire quantum register into partitions of manageable size by using segmented traps featuring loading and processor zones [3,4], and ion transfer between zones can be achieved in a fast diabatic manner optimized to reduce heating [5,6]. When increasing the complexity of such traps, planar designs are often favored, as they benefit from elaborated micro-system fabrication techniques. These allow for very flexible designs [7, 8] (for a recent review see [9]) relevant for universal quantum computing, but also in the context of quantum simulations, where customized electrode shapes can be used to realize various lattice structures and interaction types between trapped ions [10,11,12].

Ionic qubits can be manipulated with high fidelity using laser-based gates, whereas qubits encoded into hyperfine states can also be manipulated directly by radio frequency (RF) fields.

One way to maintain the addressability of single ions despite their separation being orders of magnitude below the diffraction limit is the application of a static magnetic

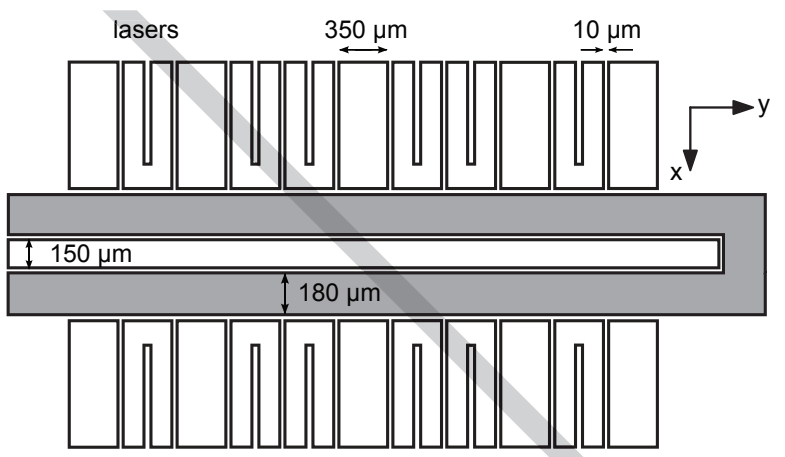

Fig. 1 Schematic of the five-electrode planar trap geometry with integrated current loops for a flexible axial magnetic gradient shape. The RF trapping field is applied to the electrodes shown in grey and provides radial trapping. The segmented dc electrodes allow for tailoring of the axial electric potential for ion transport, and, due to slots, for application of currents for a flexible axial magnetic field and gradient. The diagonal grey line indicates the direction of laser beams used for Doppler cooling and detection of trapped ions.

field gradient and exploiting an inhomogeneous Zeeman effect [13, 14, 15, 16, 17] which allows addressing in frequency space. In this way, low crosstalk can be achieved [16]. For addressing of individual ions it has also been proposed [18] and demonstrated [19] to use inhomogeneous laser fields, and addressing has been demonstrated using oscillating microwave gradients [20].

Coupling between internal and motional states of trapped ions - needed for conditional quantum dynamics with several ions - is negligible in usual ion traps when RF radiation is applied. In the presence of a static [13,15] or oscillating [21] magnetic field gradient, however, such coupling is induced. Also, coupling between spin states of different ions [14, 16, 17] arises in a spatially varying magnetic field and is thus termed magnetic gradient induced coupling (MAGIC).

A static gradient can be generated by permanent magnets [15,16] or by current loops that allow to introduce a time dependence. This was implemented into $3 \mathrm{~d}$ ion trap designs [22], discussed for planar geometries [23], and applied for 

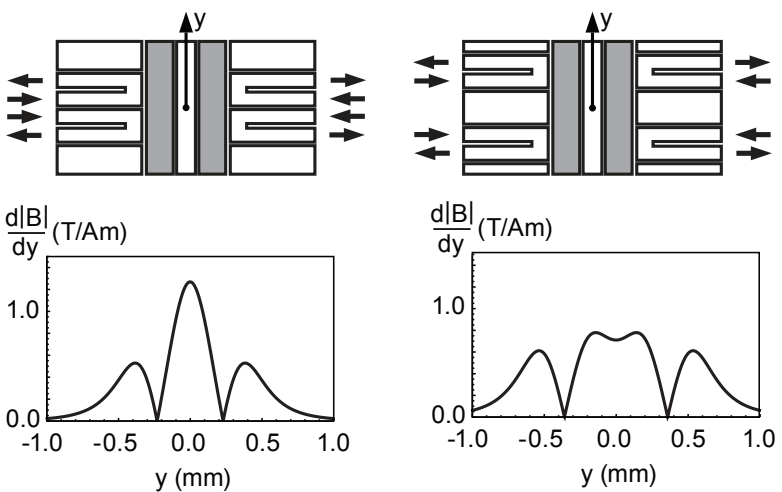

Fig. 2 Current carrying structures and magnetic field gradient of the planar ion trap. Upper panel: Electrode structure. RF electrodes are shown in grey (width of $120 \mu \mathrm{m}$ ). The ground electrode and the segmented DC electrodes (width of $350 \mu \mathrm{m}$ ) are shown in white. The DC electrodes are split allowing for the application of a current (indicated by arrows). Lower panel: Here, two possibilities to generate a magnetic field gradient are shown corresponding to the currents indicated by arrows in the upper panel. The simulated magnetic gradient (for a current of $1 \mathrm{~A}$ ) is plotted as a function of the axial coordinate $y$. Left: quadrupole configuration, right: stretched quadrupole configuration.

addressing in frequency space using a laser quadrupole transition [24]. The tailoring of the interactions between ions can be achieved by shaping the axial electrostatic trapping potential [14,25, 16], but also by changing the shape and direction of the magnetic field gradient.

In what follows, we discuss design considerations and fabrication details for a planar trap with integrated segmented loops which provide a magnetic field gradient whose spatial dependence can be tailored. We present experimental results with trapped ytterbium ions and demonstrate for the first time the application of a magnetic field gradient for RF addressing of ions in a planar trap.

\section{Experimental setup}

\subsection{Trap design and fabrication}

The trap presented here is a symmetric five-electrode planar trap design [26]. The outer dc electrodes are segmented to provide axial confinement and allow for axial ion transport. Numerical simulations based on analytical solutions for planar traps [27,28] were carried out for various electrode dimensions to maximize the trap depth at given RF amplitude, and we choose the electrode width as $180 \mu \mathrm{m}$ for the radio frequency electrodes and $150 \mu \mathrm{m}$ for the middle control electrode (see fig. 1). Eleven dc electrode pairs allow to transport ions over a range of several millimeters and define several independent trapping regions. In addition, we integrated current loops for the creation of inhomogeneous fields to allow for MAGIC.

So far, approaches for integrated current loops for five electrode planar traps designs used a current through a pat-
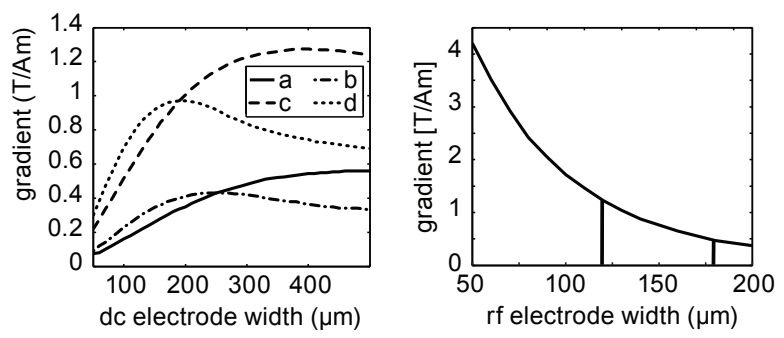

Fig. 3 Simulated axial magnetic field gradient for varying electrode widths. Left: The gradient is shown as a function of the width of the segmented dc electrode with fixed RF electrode width at $180 \mu \mathrm{m}$ (a, b) and $120 \mu \mathrm{m}(\mathrm{c}, \mathrm{d})$, respectively, for two different current configurations: quadrupole $(\mathrm{a}, \mathrm{c})$ and streched quadrupole $(\mathrm{b}, \mathrm{d})$ as motivated in fig. 2 For every fixed RF electrode width a segmented dc electrode width can be found which maximizes the gradient. Right: The gradient as a function of the RF electrode width (and in turn the trapping height) is shown; here, segmented dc electrode width is fixed at $350 \mu \mathrm{m}$. By reducing the trap dimension, gradients higher than $4 \mathrm{~T} / \mathrm{Am}$ are predicted. The RF electrode widths presented in the left part are marked by vertical lines in the right plot.

terned center wire [23,24] to create an inhomogeneous magnetic field. In that case the shape and the position of the gradient are predetermined by the design of the electrode/current loop. Here, we introduce a new approach where several segmented dc electrodes are slotted. Applying currents with individual magnitude and direction through these microstructured integrated current loops provide a magnetic field gradient with a variable shape and strength along the axial direction.

The design introduced here makes use of up to twelve segmented dc electrodes to generate the magnetic field (see fig. 1). The width of these electrodes primarily determines the shape and peak strength of the gradient for a given current. Two basic current patterns are used here to optimize the electrode/current loop geometry (compare fig. 2): in quadrupole configuration (fig. 2 left) the current is applied to any neighboring current loops in a symmetric fashion, resulting in a strong peak gradient strength for a given current. When the same pattern is applied in stretched quadrupole configuration, the gradient extends over a larger region, at the expense of a lower peak gradient strength (see fig. 2 right). The electrode width affects the peak gradient value in both scenarios and numerical simulations using Biot-Savart's law were used to find the electrode width of $350 \mu \mathrm{m}$ to be a good a compromise between the peak strengths for both patterns (see fig. 3 left). The gradient depends also on the width of the RF electrodes, as illustrated in fig. 3 showing the peak gradient strength in quadrupole configuration for a dc electrode width of $350 \mu \mathrm{m}$. A flexible shaping of the gradient can be achieved as any current pattern with varying current strength can be applied, resulting in the weighted sum of the individual gradients.

The materials chosen for the trap chip were selected for their compatibility with ultra-high-vacuum, high RF voltages and high currents up to several Ampere to obtain large trap depths and magnetic field gradients, low RF losses and high 


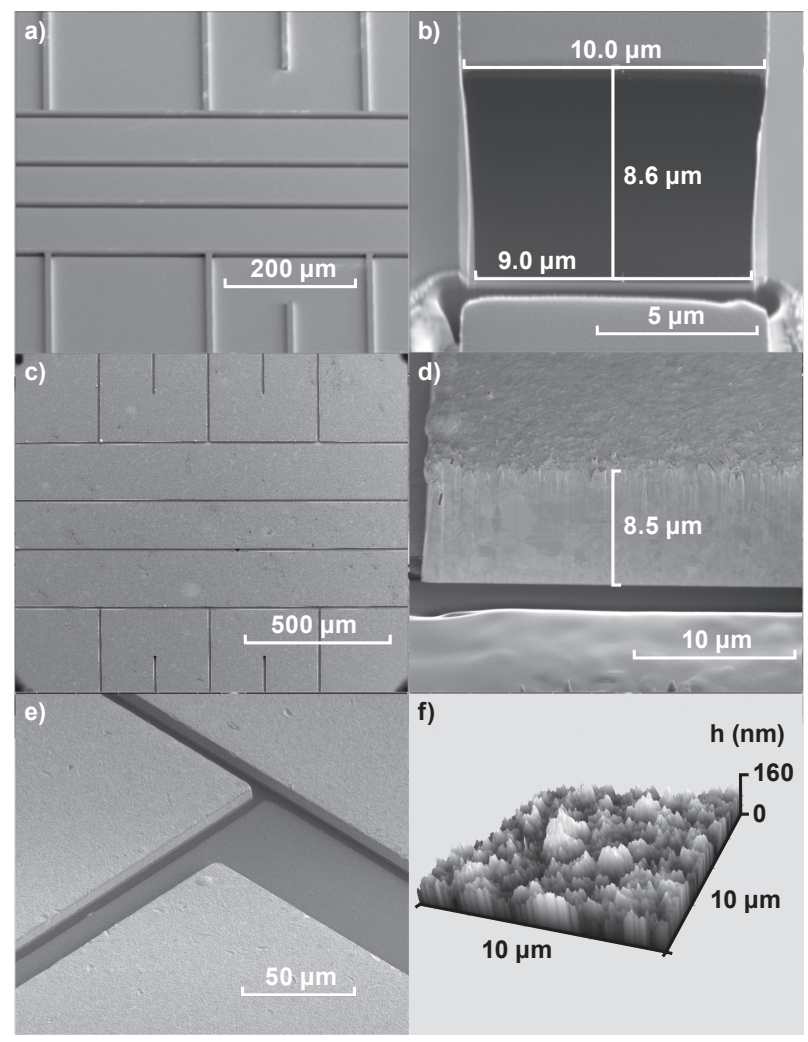

Fig. 4 Focussed-ion-beam imaging of the relevant production steps; a) Resist structure after photolithography that defines the gaps between the electrodes in the following electroplating step. In this image, the dark lines indicate resist structures that are elevated above the surface of the substrate. b) To determine the quality of the resist structure, we removed a part of the resist with an ion-beam and visualized the structure under $52^{\circ}$ relative to the substrate surface. In this way the resist thickness $(8.6 \mu \mathrm{m})$ and the widths at the top $(10 \mu \mathrm{m})$ and bottom $(9 \mu \mathrm{m})$ can be determined. c) Electroplated electrodes. d) Cut through one electroplated electrode and measured gold height to $8.5 \mu \mathrm{m}$. e) Structure after physical etching with a still existing chrome layer (dark grey) between the gold electrodes (light grey). f) A surface roughness of $20-30 \mathrm{~nm}$ rms is measured with an AFM for different chips and different positions on the chips. The figure shows one sample for illustration.

thermal conductivity to remove heat intake efficiently. The chip substrate is made of sapphire, as this material allows for high RF amplitudes due to a high electrical resistance and low absorption of RF power. Furthermore, a good surface roughness of around $3 \mathrm{~nm}$ can be obtained. The adhesion of the electrode material (in our case gold) is essential and we improve it by an additional intermediate adhesion layer of chromium. Any thermal load, either by RF losses or from high currents required for large magnetic gradients, is efficiently dissipated due to the large thermal conductivity $(45 \mathrm{~W} / \mathrm{mK})$ of sapphire.

The largest possible magnetic field gradient is given by the damage threshold of the integrated coils due to ohmic heating, so we aim to obtain a high damage threshold by having a low resistivity (using thick electrodes made of gold, which has a low specific resistivity) and by quickly removing

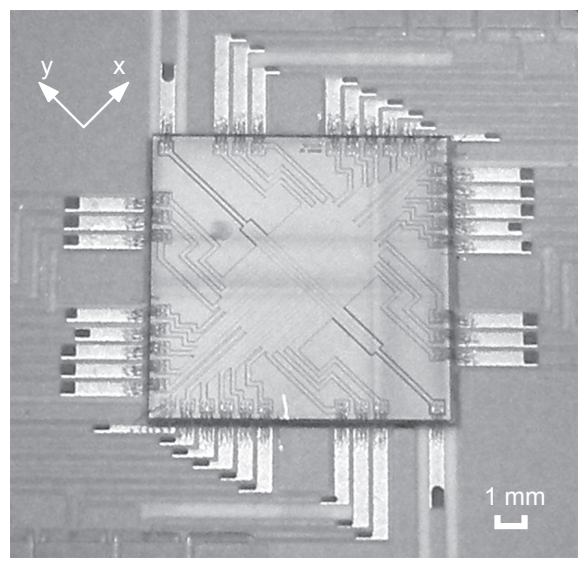

Fig. 5 Assembled electroplated ion trap chip with an edge length of $11 \mathrm{~mm}$ onto an alumina carrier with printed silver-palladium wires. Every electrode is ball bonded six times with $50 \mu \mathrm{m}$ gold wires to gold coated bond pads on the carrier.

the heat by the good thermal conductivity of the gold electrode and the sapphire substrate.

We create the trap electrodes by sputtering a $10 \mathrm{~nm}$ chrome adhesion layer followed by a $50 \mathrm{~nm}$ gold seed layer. Before every sputtering process a physical etching step cleans and smoothes the processed surface. The layer thickness obtained by standard sputtering or evaporating processes is usually limited to around $1 \mu \mathrm{m}$. Thicker structures can be obtained by electroplating. The gaps between the electrodes are defined by optical lithography (fig. 4 a,b). In this process the wafer is spin coated with negative photo resist (AZ15nXT) with a height of $8.5 \mu \mathrm{m}$. A baking step reduces the solvent before the resist is covered with a photolithography mask and exposed with uv light (i-line of $\mathrm{Hg}$ lamp). Another baking step crosslinks the resist. Then, after a chemical developer (AZ826) has removed the exposed resist, a cleaning process with oxygen plasma removes unintended residual resist. The resist structure obtained in this process yields nearly vertical edges and high aspect ratios (fig. 4 a,b). To determine the quality of the resist structure, we removed a part of the resist with an ion-beam and visualized the structure under $52^{\circ}$ relative to the surface. In this way the resist thickness and the widths at the top and the bottom can be determined. It can be seen in fig. 4 b) that these widths differ only by roughly $10 \%$ $(9 \mu \mathrm{m}$ versus $10 \mu \mathrm{m})$.

Electroplating is carried out using an open bath (Metakem SF6) under atmospheric conditions. The bath is temperature stabilized and $\mathrm{pH}$-value controlled and can operate with current densities as low as the minimum specified value for the solution $\left(1 \mathrm{~mA} / \mathrm{cm}^{2}\right)$. At this current density, we obtain a gold deposition rate around $60 \mathrm{~nm} / \mathrm{min}$ and a smooth surface quality with an rms roughness around $25 \mathrm{~nm}$ (see fig. 4 f). We electroplate gold layers up to a thickness of $8.5 \mu \mathrm{m}$ (see fig. 4 $\mathrm{c}, \mathrm{d})$. The resist is removed after electroplating using wet etching (DMSO) before the seed layers can be physically etched with an argon plasma (fig. 4 e) which can be controlled on a nanometer scale. 
The trap is mounted on a custom made chip carrier made of alumina for its high thermal conductivity of $25 \mathrm{~W} / \mathrm{mK}$ and its machinability with pulsed $\mathrm{CO}_{2}$ or Nd:YAG lasers. We use thick film technology [29] to print wires, resistors and capacitors onto the chip carrier to integrate low pass filters for each $\mathrm{dc}$ electrode with an cut-off frequency in the $\mathrm{kHz}$ range. Similar chip carriers have been demonstrated before and can also be used as a vacuum interface [22]. The maximum current is at present limited by the resistance of the feed wires on the carrier which is near $8 \Omega$ for a single loop.

The trap depth can be increased by mounting a conductive mesh at a distance of a few millimeters parallel to the trap surface and applying a positive voltage [30]. Such an electrode also reduces the effect of stray charges of the optical viewports used for the detection of the ion (see sec. 2.2). Here, we use instead a glass slide made of borosilicate glass with a thickness of $60 \mu \mathrm{m}$ and coat it with $100 \mathrm{~nm}$ layer of transparent, but conductive indium-tin-oxide (ITO) [31] by sputtering. In this way, the glass slide can be connected to a voltage supply and can be used as a transparent electrode (transmission $70 \%$ at $369 \mathrm{~nm}$ ).

\subsection{Laser system and detection}

The laser system is, apart from minor modifications, as it has been used to trap ions in a $3 \mathrm{~d}$ segmented linear trap with a built-in magnetic gradient coil and is described in [22]. All lasers are external cavity diode lasers, locked to temperature and pressure stabilized low drift medium finesse FabryPerot cavities (with finesses in the range of 50...200). The lasers are fibre coupled and overlapped using dichroic mirrors before they enter the vacuum chamber. All wavelengths are simultaneously determined using a home-built scanning Michelson interferometer which allows for a relative accuracy of $\delta \lambda / \lambda \approx 10^{-8}$ corresponding to a few tens of $\mathrm{MHz}$ for all our lasers. Using this lambdameter alone, one can set the wavelengths precisely enough to see ionic fluorescence. A beam of neutral atoms is generated by ohmic heating of a miniaturized atomic oven. The atoms are photoionized using two-step photo-ionization with a resonant first step which is driven using a laser near $398 \mathrm{~nm}[32,33,34]$. From there, the cooling laser (see below) near $369 \mathrm{~nm}$ drives the transition into the ionization continuum. The laser beams are aligned parallel to the trap surface and are adjusted under $45^{\circ}$ relative to the trap axis to achieve Doppler cooling of radial and axial modes. The out-of-plane motion is not or only weakly cooled due to fringe potentials.

The relevant energy levels of ${ }^{172} \mathrm{Yb}^{+}$are shown in fig. 6 For cooling and state-detection, we use the resonance transition between the $S_{1 / 2^{-}}$and the $P_{1 / 2^{-}}$state near $369 \mathrm{~nm}$. Spontaneous decay into the $D_{3 / 2}$-state requires an additional laser near $935 \mathrm{~nm}$ for repumping into the ground state $S_{1 / 2}$. Collisions with background gas with sufficient energy can mix the $D_{3 / 2}$-state with the $D_{5 / 2}$-state. This state can decay into the $F_{7 / 2}$-state which has been used in clock experiments and has a predicted unperturbed lifetime of several
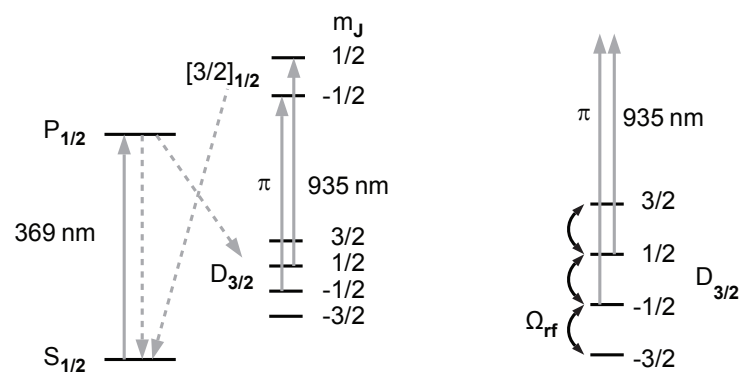

Fig. 6 Relevant energy levels of ${ }^{172} \mathrm{Yb}^{+}$(not to scale). Left: The electric dipole transition between the $\mathrm{S}_{1 / 2}$ ground state and the $\mathrm{P}_{1 / 2}$ excited state near $369 \mathrm{~nm}$ is used for Doppler cooling and state selective detection by detecting resonance fluorescence with a photomultiplier or an intensified CCD camera (termed "cooling fluorescence"). Laser light near $935 \mathrm{~nm}$ coupling the metastable state $\mathrm{D}_{3 / 2}$-state to the $[3 / 2]_{1 / 2}$ state allows for control of optical pumping into the $\mathrm{D}_{3 / 2}$-state. Right: $\mathrm{RF}$ radiation $\left(\Omega_{\mathrm{rf}}\right)$ couples the states populated by optical pumping to those which are depopulated by the repumping laser near $935 \mathrm{~nm}$ (see sec. 4).

years [35]. Considering the background pressure in our experiments $<3 \cdot 10^{-11}$ mbar, this collision-assisted loss rate is in the range of sub-milli Hertz and thus we do not repump this state with an additional laser but in such cases drop the ion from the trap and reload.

Fluorescence from trapped ions is collected with a large numerical aperture lens-system $(\mathrm{NA}=0.4)$ which is optimized for diffraction limited imaging of ions over a large field of view [36]. A schematic cross section of the light gathering system can be found in [22]. The fluorescence is discriminated against stray-light from the trap chip by a telecentric imaging system. This setup located in an aluminum box anodized for high absorption $(\approx 90 \%$ absorption for $369 \mathrm{~nm}$ laser light) includes three planes where high absorption coated moveable razor blades ( $95 \%$ absorption for $369 \mathrm{~nm}$ laser light) are mounted. Two blade pairs form a rectangular aperture localized in the focal plane of the imaging objective. Ions are imaged via an extension lens onto an EMCCD camera (Andor iXon ${ }^{+}$). A third pair of blades aid in blocking light scattered from objects originating at different locations near the trapping region. Thus, the signal-tobackground ratio can be improved. Stray-light from all lasers with wavelengths different from $369 \mathrm{~nm}$ is effectively suppressed using a narrow band-pass filter with a spectral width of $6 \mathrm{~nm}$ (FWHM) in front of the camera.

\subsection{Electrical signals}

The RF voltage required to trap ions is generated by a signal generator which is amplified and fed into a helical resonator and the details of the setup are discussed in this section. The helical resonator follows the general concept reported in [37, 38, 39] and is designed as an autotransformer. This approach yields lower insertion loss, but also results in lower Q-factors compared to the approach described in [40]. We carefully designed the resonator for mechanical stability and wound the 


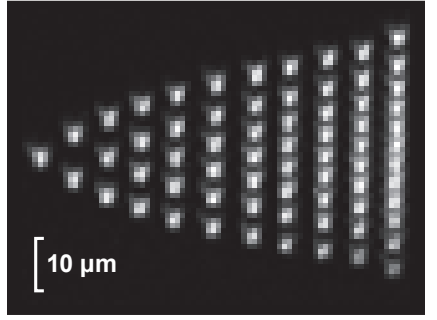

Fig. 7 A composite picture of trapped ${ }^{172} \mathrm{Yb}$ ion chains. Between one (leftmost columns) and twelve (rightmost column) ions are trapped. Each of the twelve individual ion chain pictures is taken from one single loading procedure.

helix on a threaded low loss dielectric tube (PTFE). The mechanical stability results in a low drift of the resonance frequency of $\pm 30 \mathrm{~Hz}$ over several hours. This was measured using a capacitive load $(\approx 30 \mathrm{pF})$ which is comparable to our trap including connectors $(\approx 35 \mathrm{pF})$. The insertion point of the primary coil, which is critical for impedance matching, is realized as a slider which can be firmly fixed with a set screw with good electrical contact, but, at the same time, can be moved with little effort. The tube can be filled, also partially, by a dielectric to tune the resonance frequency of the circuit. The frequency tuning range is found to be on a percent scale of the resonance frequency (for up to $80 \%$ filling with PTFE) and can alternatively be achieved by varying the load, for instance, by a different length of the cable connecting the resonator to the trap.

Even with a Q-factor near 80 we find that, due to low insertion losses, the resonator can drive a trap like the one described in [22] with a RF power of only $0.25 \mathrm{~W}$, which is readily delivered by the signal generator (as, for example, a Rigol DG1012) and requires no further amplification. In the experiments presented here, the RF amplitude is generated by a frequency generator (Hameg HM8032) and amplified with a Kalmus $110 \mathrm{C}$ by $40 \mathrm{~dB}$ to a power of approximately $0.4 \mathrm{~W}$. The helical resonator boosts the peak-to-peak-voltage at the trap frequency of $14.7 \mathrm{MHz}$. The voltage is fed into the trap and simultaneously monitored via a $1 \mathrm{pF}$ capacitance probe. The system is optimized to avoid ground loops.

A system of DAC cards (Adwin Pro II) connected to $50 \Omega$ drivers delivers 10 tunable voltages in the range of $\pm 10 \mathrm{~V}$. Via jumpering (compare [22]) up to 75 potentials can be routed to the trap electrodes via sub-d connectors.

\section{Trap characteristics}

We demonstrate trapping ${ }^{172} \mathrm{Yb}^{+}$ions in our planar trap (fig. 7). The storage time with laser cooling but without repumping the $F_{7 / 2}$-state is several hours for single ions and several 10 minutes for ion chains up to 10 ions.

A measured trapping height of $(160 \pm 10) \mu \mathrm{m}$ is in agreement with the numerical simulation of the trapping potential. The measured ion-ion distance for two ions is $10 \mu \mathrm{m}$ (taking into account the independently determined magnification
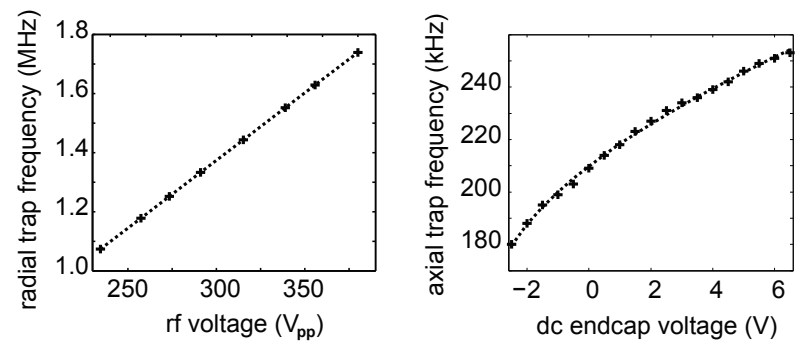

Fig. 8 Measured trap frequencies as a function of applied voltages. Left: Measured radial trap frequency which is directed parallel to the trap surface with $1 \mathrm{~V}_{\mathrm{dc}}$ applied to the endcap electrodes. Right: Measured axial trap frequency at $250 \mathrm{~V}_{\mathrm{pp}} \mathrm{RF}$ amplitude. The errors for measured frequencies are $0.5 \mathrm{kHz}$ and for applied voltages $0.01 \mathrm{~V}_{\mathrm{dc}}$ and $1 \mathrm{~V}_{\mathrm{pp}}$ respectively.
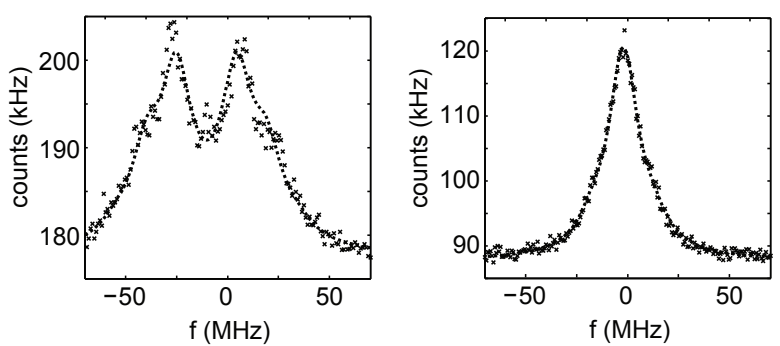

Fig. 9 Micromotion compensation analysed with absorption spectra of the $935 \mathrm{~nm}$ repump laser (see text). Left: Before micromotion compensation several motional sidebands at multiples of the trap drive frequency of $14.7 \mathrm{MHz}$ are visible. Right: Micromotion sidebands are suppressed in the spectrum after micromotion compensation was carried out and predominantly the carrier is visible. The function fitting the dataset is given by the lineshape calculated for an oscillating ion [41]. The variations in frequency and amplitude between the two spectra shown here are caused by laser frequency drifts during data taking.

of the detection system). Ions are stable for RF peak-to-peak amplitudes between $150 \mathrm{~V}_{\mathrm{pp}}$ and $400 \mathrm{~V}_{\mathrm{pp}}$. With a typical trapping amplitude of $250 \mathrm{~V}_{\mathrm{pp}}$ and a trap drive frequency of 14.7 MHz, the stability parameter [26]

$$
q=\frac{2 Q V_{\mathrm{rf}}}{m \Omega^{2} r_{0}^{2}}
$$

is determined to be 0.22 , with the charge $Q$, RF amplitude $V_{\mathrm{rf}}=V_{\mathrm{pp}} / 2$, trap drive frequency $\Omega$ and trap geometry factor $r_{0}$. The trap depth [26]

$$
\Psi_{0}=\frac{Q^{2} V_{\mathrm{rf}}^{2}}{4 m \Omega^{2} r_{0}^{2}}
$$

is determined to be $73 \mathrm{meV}$.

We measure trap frequencies by resonant heating, which occurs, when the trap frequency coincides with the frequency of a sinusoidal 'tickling' signal applied to one dc electrode. The motional frequencies are determined for the axial direction in the range from $180 \mathrm{kHz}$ to $250 \mathrm{kHz}$ (fig. 8 left) and for the radial direction parallel to the trap surface between 1.0 $\mathrm{MHz}$ and 1.8 MHz (fig. 8 right). 
Stray fields may prevent the ion from being trapped at the bottom of the effective potential, where the RF electric field vanishes. In that case the ion's driven motion results in sidebands in the absorption spectrum that are separated from the carrier by multiples of the RF trap drive frequency (fig.9 9 left). To detect and compensate this motion, the dependence of the ion fluorescence intensity on the detuning of the $935 \mathrm{~nm}$ laser is analyzed. By changing potentials applied to the segmented dc electrodes the ion can be moved slightly along the radial direction towards the RF minimum, where the sidebands are reduced and the carrier dominates the absorption spectrum (fig. 9 right).

Background light is reduced by a telecentric imaging setup as described above. The signal-to-background-ratio is optimized starting with the blades initially fully open and then closing them until the best ratio is achieved. For small apertures, both signal, and background depend approximately linearly on the area of the aperture and we use the intersection of the tangent to the fluorescence rate with the fluorescence rate which saturates for large apertures to find a working point for the blade setting yielding a signal-to-background-ratio of $211 \pm 9$ compared to $54 \pm 2$ with fully open blades.

\section{RF-optical double resonance spectroscopy}

We demonstrate one of the two basic effects of an inhomogeneous magnetic field, the addressing of ions in frequency space, using the Zeeman levels of the $D_{3 / 2}$ manifold (see fig. 6). These levels have been previously used to show addressability of ions using RF transitions in a magnetic field gradient [15].

The metastable $\mathrm{D}_{3 / 2}$-state (lifetime of $52.2 \mathrm{~ms}$ [43]) is populated by spontaneous decay from the $P_{3 / 2}$-state with a branching ratio of approximately $0.5 \%$ [42]. Thus optical pumping into this level occurs on a microsecond time-scale into all Zeeman levels. In order to close the fluorescence and cooling cycle, laser light near $935 \mathrm{~nm}$ is applied that excites the ion to the $[3 / 2]_{1 / 2}$-state which subsequently decays to the ground electronic state. For cooling and detection, the polarization of the $935 \mathrm{~nm}$ light has to contain at least $\sigma^{+}$and $\sigma^{-}$components in order to prevent optical pumping into any of the Zeeman states of the $\mathrm{D}_{3 / 2}$-level. Light near $935 \mathrm{~nm}$ containing $\pi, \sigma^{+}$, and $\sigma^{-}$components is achieved by using a linearly polarized light beam incident on the ion at $45^{\circ}$ relative to the quantization axis. Thus, the population from all Zeeman substates is pumped back to the ground electronic state. However, in order to demonstrate individual addressing we want to first prepare an ion deterministically in one of the Zeeman substates of the $D_{3 / 2}$-level by optical pumping. This process is described in what follows.

\subsection{State initialization}

When the repumper near $935 \mathrm{~nm}$ is linearly polarized and its electric field is aligned parallel to the magnetic field that

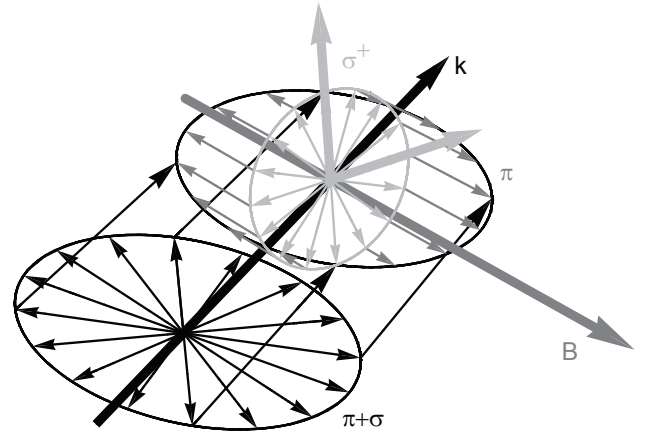

Fig. 10 Decomposition of suitable elliptical polarization into phase coherent linear and circular polarizations. If the projection of the elliptical polarization onto the plane perpendicular to the external field determining the quantization axis (B) yields exactly a circle, the total polarization will only drive a $\pi$ and one $\sigma$ component.

determines the quantization axis, it will exclusively drive $\pi$ transitions which do not change the Zeeman quantum number $m_{J}$. Thus, population accumulates in the Zeeman states $m_{J}= \pm 3 / 2$ that are not coupled to the light field, since the $[3 / 2]_{1 / 2}$-state cannot be accessed from them by $\pi$-transitions. Both levels $m_{J}= \pm 3 / 2$ are populated with equal probability (assuming no imperfections in the polarization state). Once the ion is optically pumped into these two Zeeman states, fluorescence and cooling of the ion stops. This initialization scheme requires an electric field polarization parallel to the magnetic field, and thus a propagation direction, indicated by the $k$-vector of the light field, perpendicular to it.

When one circular polarization component is added to the $935 \mathrm{~nm}$ light, population will be trapped in a single Zeeman state, for instance in the $m_{J}=3 / 2$-state for $\sigma_{+}$-polarization (compare fig. 6). Circular polarization requires a field vector rotating around the magnetic field and thus requires a $k$ vector parallel to this field.

Instead of using two light fields for initializing the ion in one Zeeman state, we construct the polarization state of the field for a given $k$-vector, as shown in fig. 10. In this way the qubit can be initialized in a single Zeeman state using only one laser beam. This is a general approach, because of its independence on the direction of the magnetic field that determines the quantization axis.

Here, we arrange the degree of elliptical polarization such, that the projection onto the plane normal to the magnetic field is circular. In this way, the total light field is composed of a linearly polarized electric field parallel to the magnetic field and a circularly polarized component perpendicular to the magnetic field. Thus, population can be trapped in either one of the Zeeman states $m_{J}= \pm 3 / 2$ (creating a "dark" state).

\subsection{RF manipulation of the Zeeman states}

Individual addressing of ions is demonstrated using RFoptical double resonance spectroscopy on the $\mathrm{D}_{3 / 2}$-state. The 
ion is first prepared in a desired Zeeman state as described above (a dark state). Then RF radiation is applied that brings the ion back into the fluorescence cycle, if it's frequency is close to the resonance that corresponds to a transition between Zeeman substates. This resonance frequency is determined by the strength of the local magnetic field that the ion is exposed to as explained in what follows.

The degeneracy of its Zeeman-manifold is lifted by a magnetic field $B=B_{0}+y \partial_{y} B_{G}$ composed of an offset field $B_{0}$ and an additional field with constant gradient $\partial_{y} B_{G}$ (the axial direction $\mathbf{y}$ is the direction of the softest trap frequency).

The magnitude of $B$ determines the resonance frequency of magnetic dipole transitions between the Zeeman states. The (linear) Zeeman shift $\Delta E_{J}$ by a magnetic field $B$ is given by $\Delta E_{J}=g_{J} m_{J} \mu_{B} B$ with Landé g-factor $g_{J}$, magnetic quantum number $m_{J}$, and the Bohr magnetron $\mu_{B}$. Magnetic dipole transitions between levels with $\Delta m_{J}= \pm 1$ (magnetic $\sigma$-transition) with resonance frequency

$$
f=\frac{g_{J} \mu_{B} B}{h}
$$

are driven using an RF field that is generated by a dipole coil wrapped around the light gathering optics. The magnitude of $B_{0}$ was chosen to be about $0.6 \mathrm{mT}$ resulting in a resonance frequency $f \approx 7 \mathrm{MHz}$.

We generate the RF signal using a signal generator (VFG 150). This device allows to control the frequency, amplitude and phase and allows for fast phase continuous and phase coherent switching [44, 45,46]. The output of the signal generator is amplified (Kalmus 110C) with a gain of $40 \mathrm{~dB}$ to a power of up to 5 Watts. The signal is applied with a loop antenna, which forms a resonance circuit with a series capacitor. Impedance matching is achieved by using an adjustable series resistance.

The quantization axis is defined by the field of a neodymium permanent magnet positioned at a distance around $150 \mathrm{~mm}$ from the trap center, which leads to a magnetic offset field near $0.6 \mathrm{mT}$. RF-optical double resonance spectra, as shown in fig. 11 13 are taken using the following procedure: the repumper is split, manipulated with a half wave plate and recombined using polarizing beam splitters. A half wave and a quarter wave plate after the recombining polarizing beam splitter (PBS) are adjusted such, that optical pumping is achieved using light only from the first arm with an elliptical polarization as described in sec. 4.1. Additional light from the second arm, which counteracts optical pumping is chopped with a chopper-wheel to switch between cooling cycles (no optical pumping) and spectroscopy cycles. During spectroscopy cycles, the cooling laser and the repumper (leading to optical pumping), RF manipulation and detection by the camera are simultaneously active, leading to incoherent RF-optical double resonance spectra with Lorentzian line shapes.

The amplitude and the width of the spectral lines are determined by the power of the repumper laser and the RF radiation. Fig. 11 shows amplitude normalized spectra obtained

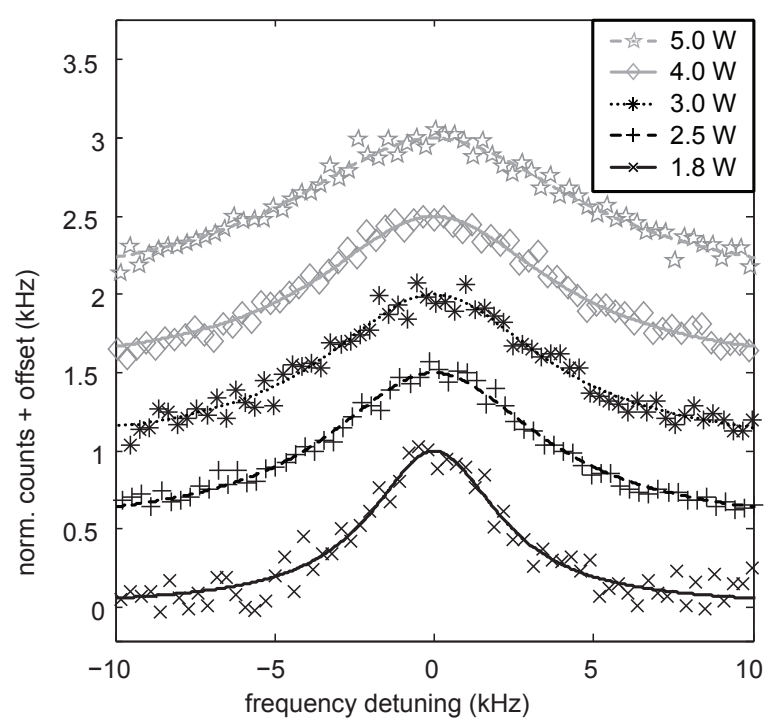

Fig. 11 RF-optical double resonance spectra. The RF power is varied while the $935 \mathrm{~nm}$ laser power remains constant near $5 \mu \mathrm{W}$. The transition width is reduced down to $5 \mathrm{kHz}$ by diminishing the RF power. A small width is necessary to detect small gradients. For a better visualization offsets are added to the displayed spectra. The spectra are normalized and fitted with Lorentzian profiles.

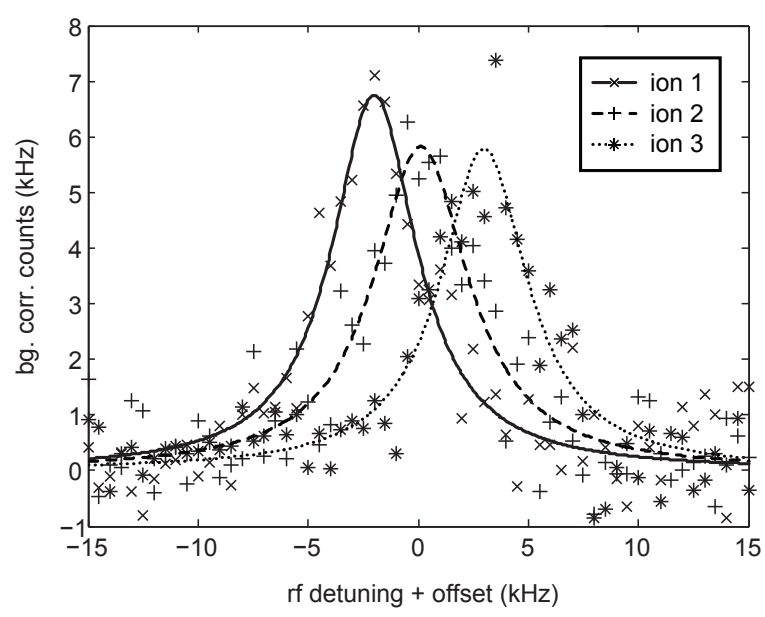

Fig. 12 RF-optical double resonance spectra of a three-ion chain. The measured gradient of $25 \mathrm{mT} / \mathrm{m}$ is composed of the gradient generated by a current loop pair $(14 \mathrm{mT} / \mathrm{m})$ and the one due to the inhomogeneous, external magnetic field $(11 \mathrm{mT} / \mathrm{m})$. Lorentzian functions fit the measured, background corrected data points.

by scanning the RF frequency with constant laser power near $5 \mu \mathrm{W}$ and varying RF power. For an RF power around $1.8 \mathrm{~W}$ we find the width of the resonance to be around $5 \mathrm{kHz}$ (FWHM). Under such conditions, the narrow width of the resonance allows for a sensitive detection of changes in the magnetic field strength (by changing the current) or its inhomogeneity. The resonance frequency of the $\sigma$-transition is given by eq. 3 and can thus be changed by a variation of $B$ at the position of the ion. This was checked by sending a cur- 


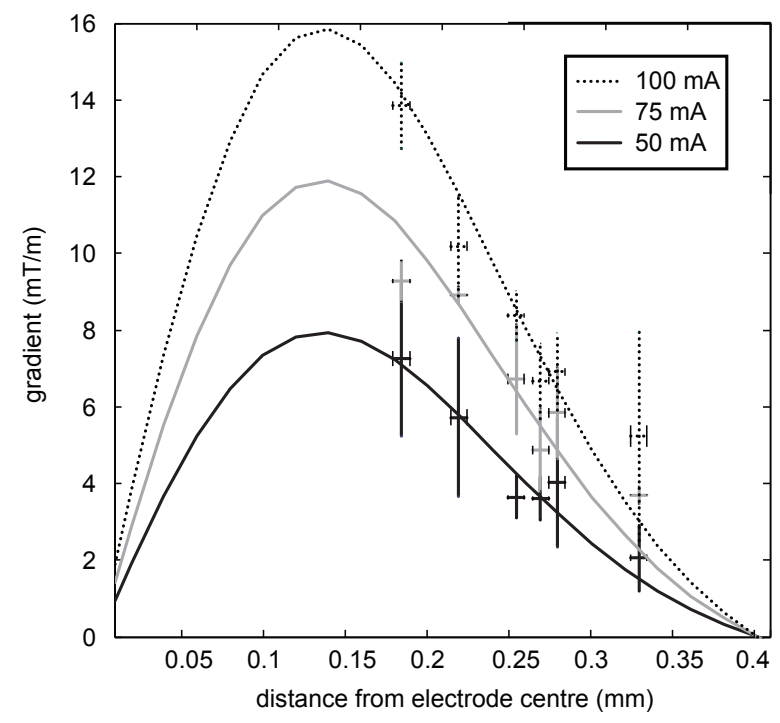

Fig. 13 Measured magnetic field gradients with currents at $50 \mathrm{~mA}$, $75 \mathrm{~mA}$ and $100 \mathrm{~mA}$ as a function of ion position. The lines indicate the results of numerical simulations with no free parameters taking into account the additional inhomogeneity from the permanent magnet mounted outside the vacuum chamber. The abscissa shows the distance from the centre of the current carrying electrode pair. The error bars are statistical errors from a set of up to 30 single measurements.

rent through the dc electrodes and mapping out the resonance frequency as a function of the applied current.

The magnetic field to which a given ion is exposed depends on its position, and for a three-ion chain a small gradient will result in three resonances whose center frequencies are shifted with respect to each other. To demonstrate this, we send a current of up to $100 \mathrm{~mA}$ through two coils of the same segment and observe a splitting as shown in fig. 12 From this splitting and a measurement of the ion separation, the gradient can be determined. The ion separation is either directly determined from EMCCD images and the calibrated magnification of the imaging system, or by measuring the axial trap frequency and using the approximation for harmonic potentials

$$
\Delta y_{2}=\sqrt[3]{\frac{q^{2}}{2 \pi \epsilon_{0} m \omega_{\mathrm{cm}}^{2}}} \quad \Delta y_{3}=\sqrt[3]{\frac{5}{8}} \Delta y_{2}
$$

( $\Delta y_{2}$ being the separation between two ions in a twoion chain and $\Delta y_{3}$ being the separation between two ions in a three-ion chain with centre-of-mass frequency $\omega_{\mathrm{cm}}$ ). Numerical simulations with no free parameters and currents of $50 \mathrm{~mA}, 75 \mathrm{~mA}$ and $100 \mathrm{~mA}$ yield an expected maximum gradient of $16 \mathrm{mT} / \mathrm{m}$ and agree well with the measurements carried out as discussed here for various currents and positions of the ion string (see fig. 13,
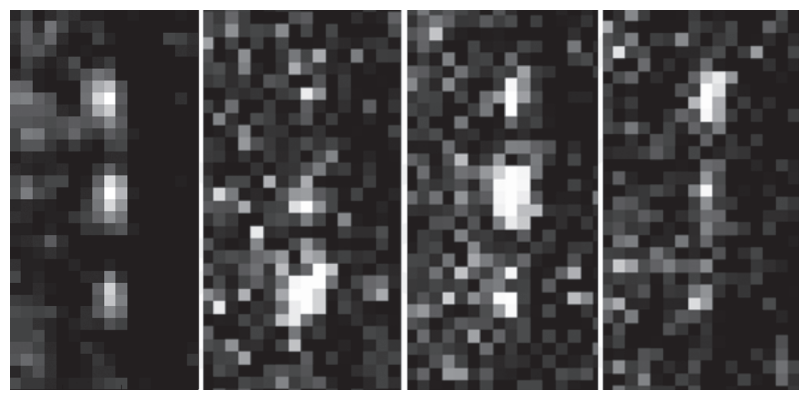

Fig. 14 Addressing an ion chain of three ions in frequency space by tuning the applied RF frequency. The achieved separation in frequency space is near $2.6 \mathrm{kHz}$ with a current amount of $100 \mathrm{~mA}$ through an electrode pair adding to an offset gradient generated by an external neodymium magnet.

\subsection{RF addressing}

With counter propagating currents of $100 \mathrm{~mA}$ through a current loop pair and the additional inhomogeneity from the permanent magnet a splitting of about $2.6 \mathrm{kHz}$ is achieved near the maximal gradient position of $130 \mu \mathrm{m}$ from the segment electrode centre for axial trapping frequencies near $200 \mathrm{kHz}$ in a three-ion chain. This splitting is sufficient to individually address ions as is demonstrated here for three ions in fig. 14 . Using the average peak width of approximately $5 \mathrm{kHz}$ from the measured data presented in fig. 12, we calculate the maximum undesired excitation probability $p$ of a neighboring ion as $0.48 \pm 0.01$ corresponding to a addressing fidelity of $F=1-p_{\max }=0.52 \pm 0.01$ where $p_{\max }$ is the maximum unintended excitation probability of all other ions. The corresponding fidelity for a chain of two ions, under similar conditions would be $0.59 \pm 0.01$, owing to the larger ion separation. This value can be increased by applying a larger gradient, using lower trapping frequencies, or by narrowing down the width of the resonance by applying less RF and laser power. The constraints on the laser power can be lifted entirely, when carrying out the RF-optical double resonance spectroscopy in a coherent manner, such that the laser is switched off during RF manipulation.

\section{Conclusion and outlook}

We have discussed the design and production details for a segmented planar trap with current loops that allow for tailoring of the axial magnetic field gradient as well as the trapping potential and demonstrated trapping of ytterbium ions in such a trap. The gradient was demonstrated by applying RFoptical double resonance spectroscopy, and a first demonstration of RF addressing in a planar trap was shown. The size of the gradient was sufficient to carry out proof-of-principle experiments. For quantum information processing, however, a larger gradient will be necessary, as was demonstrated in [16, 17,24]. An obvious solution will be to apply higher currents. When currents are increased, the ultimate limit for the current is the damage threshold of the current loops (with the present trap this regime has not yet been explored). 
When increasing the current sent through the loop structures, heat management and thus cooling of the chip carrier becomes increasingly important. The dissipated heat from ohmic losses in the carrier limit at present possible currents to $100 \mathrm{~mA}$, when restricted to $0.25 \mathrm{~W}$ power applied to the structure. The power limit of the present setup was determined by analyzing the carrier temperature up to approximately $2 \mathrm{~W}$ of applied heating power, allowing for gradients near $60 \mathrm{mT} / \mathrm{m}$ with constant currents.

Pulsed currents, applied for short times, can create much larger peak magnetic field gradients compared to gradients generated by continuous currents while keeping the average power deposition low. Possible peak gradients on the order of $1 \mathrm{~T} / \mathrm{m}$ can be expected with this trap setup.

As shown in the initial considerations of this paper, higher gradients for a fixed current can be reached by reducing the trap dimensions, and from simulations we expect a gradient of up to $4 \mathrm{~T} / \mathrm{Am}$. The resulting gradient can be boosted up to $40 \mathrm{~T} / \mathrm{m}$ when applying pulsed currents of several Amperes with reduced trap dimensions, which is on the order of present magnetic field gradients in macroscopic traps using external magnets [15, 16]. With such large gradients, not only addressing with high fidelity can be achieved, but also the direct measurement of an effective spin-spin coupling, and its application in quantum information science becomes possible [16].

\section{Acknowledgments}

We would like to acknowledge M. Epping for trap simulations, M. Böhm, D. Schäfer-Stephani, K. Watty, A. Bablich, P. Haring-Bolivar, H. Schäfer, E. Ilichev, B. Ivanov, and S. Zarazenkov for their support during chip production, D. Gebauer, D. Junge, and A. H. Walenta for the production of the chip carrier, our electrical and mechanical work shops, and especially S. Spitzer for his support regarding all electronics, and T. Collath, T. F. Gloger, D. Kaufmann and P. Kaufmann for providing the laser system used in this work.

We acknowledge funding by the Bundesministerium für Bildung und Forschung (FK 01BQ1012), and from the European Community's Seventh Framework Programme (FP7/2007-2013) under Grant Agreement No. 270843 (iQIT) and No. 249958 (PICC).

${ }^{\dagger}$ Present address: Max-Born-Institut für Nichtlineare Optik und Kurzzeitspektroskopie, 12489 Berlin, Germany

\section{References}

1. M. A. Nielsen and I. L. Chuang: Quantum Computation and Quantum Information (Cambridge U.P.)

2. R. Blatt and D. J. Wineland: Nature 45, 1008-1015 (2008).

3. D. J. Wineland, C. Monroe, W. M. Itano, D. Leibfried, B. E. King, and D. M. Meekhof: J. of Res. NIST, 103, 259 (1998).

4. D. Kielpinski, C. Monroe, and D. Wineland: Nature 417, 709712 (2002)
5. A. Walther, F. Ziesel, T. Ruster, S. T. Dawkins, K. Ott, M. Hettrich, K. Singer, F. Schmidt-Kaler, and U. Poschinger: Phys. Rev. Lett. 109, 080501 (2012).

6. R. Bowler, J. Gaebler, Y. Lin, T. R. Tan, D. Hanneke, J. D. Jost, J. P. Home, D. Leibfried, and D. J. Wineland: Phys. Rev. Lett. 109, 080502 (2012).

7. S. Seidelin, J. Chiaverini, R. Reichle, J. J. Bollinger, D. Leibfried, J. Britton, J. H. Wesenberg, R. B. Blakestad, R. J. Epstein, D. B. Hume, W. M. Itano, J. D. Jost, C. Langer, R. Ozeri, N. Shiga, and D. J. Wineland: Phys. Rev. Lett. 96, 253003 (2006).

8. C. Pearson, D. Leibrandt, W. Bakr, W. Mallard, K. Brown, and I. Chuang: Phys. Rev. A 73, 032307 (2006).

9. M. D. Hughes, B. Lekitsch, J. A. Broersma, and W. K. Hensinger: Cont. Phys., Vol. 52, No. 6, 505-529 (2011).

10. J. H. Wesenberg: Phys. Rev. A 78, 063410 (2008).

11. R. Schmied, J. H. Wesenberg, and D. Leibfried: Phys. Rev. Lett. 102, 233002 (2009).

12. J. D. Siverns, S. Weidt, K. Lake, B. Lekitsch, M. D. Hughes, and W. K. Hensinger: New J. Phys. 14, 085009 (2012).

13. F. Mintert and C. Wunderlich: Phys. Rev. Lett. 87, 25 (2001).

14. Ch. Wunderlich: in Laser Physics at the Limit (Springer, New York, 2002)

15. M. Johanning, A. Braun, N. Timoney, V. Elman, W. Neuhauser, and C. Wunderlich: Phys. Rev. Lett. 102, 073004 (2009).

16. A. Khromova, C. Piltz, B. Scharfenberger, T. F. Gloger, M. Johanning, A. F. Varón, and C. Wunderlich: Phys. Rev. Lett. 108, 220502 (2012).

17. C. Piltz, B. Scharfenberger, A. Khromova, A. F. Varón, and C. Wunderlich: Phys. Rev. Lett. 110, 200501 (2013)

18. P. Staanum and M. Drewsen: Phys. Rev. A 66, 040302 (2002).

19. N. Navon, S. Kotler, N. Akerman, Y. Glickman, I. Almog, and R. Ozeri: arXiv:1210.7336 2 [quant-ph].

20. U. Warring, C. Ospelkaus, Y. Colombe, R. Jördens, D. Leibfried, and D. J. Wineland: Phys. Rev. Lett. 110, 173002 (2013)

21. C. Ospelkaus, U. Warring, Y. Colombe, K. R. Brown, J. M. Amini, D. Leibfried, and D. J. Wineland: Nature 476, 181 (2011).

22. D. Kaufmann, T. Collath, M. T. Baig, P. Kaufmann, E. Asenwar, M. Johanning, and C. Wunderlich: Appl. Phys. B 107, 935-943 (2012).

23. J. Welzel, A. Bautista-Salvador, C. Abarbanel, V. WinemanFisher, Ch. Wunderlich, R. Folman, and F. Schmidt-Kaler: Eur. Phys. J. D 65, 285-297 (2011).

24. S. Wang, J. Labaziewicz, Y. Ge, R. Shewmon, and I. Chuang: Appl. Phys. Lett. 94, 094103 (2009).

25. H. Wunderlich, C. Wunderlich, K. Singer, and F. SchmidtKahler: Phys. Rev. A 79, 052324 (2009).

26. J. Chiaverini, R. Blakestad, J. Britton, J. Jost, C. Langer, D. Leibfried, R. Ozeri, and D. Wineland: Quantum Inf. and Comp., Vol. 65, No. 6, 419-439 (2005).

27. M. H. Oliveira and J. A. Miranda: Eur. J. Phys. 21, 31 (2001).

28. M. G. House: Phys. Rev. A 78, 033402 (2008).

29. T. K. Gupta: Handbook of Thick- and Thin-Film Hybrid Microelectronics (Wiley, New York 2005)

30. K. Brown, R. Clark, J. Labaziewicz, P. Richerme, D. Leibrandt, and I. Chuang: Phys. Rev. A 75, 15401 (2007).

31. X. Yan, F. Mont, D. Poxson, M. Schubert, J. Kim, J. Cho, and E. Schubert: Jap. J. Appl. Phys. 48, 120203 (2009). 
32. Chr. Balzer, A. Braun, T. Hannemann, Chr. Paape, M. Ettler, W. Neuhauser, and Chr. Wunderlich: Phys. Rev. A 73, 041407 (Rapid Comm.) (2006).

33. M. Johanning, A. Braun, D. Eiteneuer, C. Paape, C. Balzer, W. Neuhauser, and C. Wunderlich: Appl. Phys. B 103, 327338 (2011).

34. Y. Onoda, K. Sugiyama, M. Ikeda, and M. Kitano: Appl. Phys. B. 105, 729-740 (2011).

35. P. J. Blythe, S. A. Webster, H. S. Margolis, S. N. Lea, G. Huang, S. K. Choi, W. R. C. Rowley, P. Gill, and R. S. Windeler: Phys. Rev. A 67, 020501 (2003).

36. C. Schneider: Master Thesis, University of Siegen (2007).

37. W. W. Macalpine and R. O. Schildknecht: Proc. IRE 47, 20992105 (1959).

38. P. Vizmuller: RF Design Guide : Systems, Circuits, and Equations (Artech House, Inc., London 1995).

39. A. I. Zverev: Handbook of Filter Synthesis (Wiley \& Sons, Inc., New York 1967).

40. J. D. Siverns, L. R. Simkins, S. Weidt, and W. K. Hensinger.: Appl. Phys. B 107, 921-934 (2012).

41. D. J. Wineland and W. M. Itano, Phys. Rev. A 20, 1521 (1979).

42. S. Olmschenk, K. C. Younge, D. L. Moehring, D. N. Matsukevich, P. Maunz, and C. Monroe: Phys. Rev. A 76, 052314 (2007).

43. C. Gerz, J. Roths, F. Vedel, and G. Werth: Z. Phys. D 8, 235 (1988).

44. Ch. Wunderlich, Th. Hannemann, T. Körber, H. Häffner, Ch. Roos, W. Hänsel, R. Blatt, and F. SchmidtKaler: J. of Mod. Opt. 54, 1541 (2007). For technical specifications see http://www.physik.unisiegen.de/quantenoptik/forschung/vfg150/index.html.en?lang=en

45. N. Timoney, V. Elman, S. Glaser, C. Weiss, M. Johanning, W. Neuhauser, and C. Wunderlich: Phys. Rev. A 77, 052334 (2008).

46. N. Timoney, I. Baumgart, M. Johanning, A. F. Varón, M. B. Plenio, A. Retzker, and Ch. Wunderlich: Nature 476, 185-188 (2011). 